\title{
Preparation and characterization of superparamagnetic iron oxide nanoparticles for magnetically guided drug delivery
}

This article was published in the following Dove Press journal: International Journal of Nanomedicine

\section{Pramod Kumar \\ Shrish Agnihotri \\ Indrajit Roy}

Department of Chemistry, University of Delhi, Delhi, India
Correspondence: Indrajit Roy Department of Chemistry, University of Delhi, Delhi I I0007, India Tel +9| 956072 |85।

Email indrajitroyII@gmail.com

\begin{abstract}
Iron oxide nanoparticles have unique magnetic properties and therefore readily respond to applied magnetic fields. Moreover, their surfaces can be used to attach active molecules via various covalent or noncovalent interactions. Thus, they can be used as drug carriers for magnetically controlled delivery to specific biological sites of interest. In the present study, we have synthesized aqueous dispersed samples of citric acid-capped iron oxide nanoparticles, and the anticancer drug doxorubicin was then linked with these superparamagnetic iron oxide nanoparticles via a simple noncovalent interaction. Our results show that the conjugated drug releases from the nanoparticles in a sustained manner. The cellular uptake of these nanoparticles was found to be substantial, although it can be further enhanced using magnetic guidance. These nanoparticles (drug free) were found to be nontoxic to cells; however, upon drug conjugation, drug-induced toxicity was observed, owing to the slow release of drug from the nanoparticles. Keywords: SPION, Dox, Dox-CA-SPION, magnetically guided delivery
\end{abstract}

\section{Introduction}

Guiding therapeutic drugs to specific diseased sites using external stimulus is a promising strategy for the targeted diagnosis and treatment of diseases. ${ }^{1-3}$ Iron oxide nanoparticles have unique magnetic properties and therefore readily respond to applied magnetic fields. ${ }^{4,5}$ Moreover, their surfaces can be used to attach active molecules via various covalent or noncovalent interactions. Thus, they can be used as drug carriers for magnetically controlled delivery to specific biological sites of interest. ${ }^{6,7}$

We have synthesized aqueous dispersed samples of citric acid-capped iron oxide nanoparticles. Transmission electron microscopy (TEM) data showed the average diameter of these monodisperse nanoparticles to be around $12 \mathrm{~nm}$; energy dispersive $\mathrm{X}$-ray dispersive spectroscopy and X-ray diffraction were then used to ascertain the crystalline nature of these particles. The anticancer drug doxorubicin (Dox) was then linked with these superparamagnetic iron oxide nanoparticles (SPIONs) via a simple noncovalent interaction. In vitro studies have shown that these nanoparticles are nontoxic to cells in culture, and did not cause hemolysis in blood samples. Finally, it was demonstrated that these nanoparticles, without and with linked Dox, can be robustly delivered to cells in culture using magnetic guidance. This magnetically enhanced delivery resulted in increased Dox-induced cytotoxicity in the treated cells. These observations underscore the potential of these nanomaterials in externally targeted diagnostics and drug delivery. 


\section{Materials and methods}

Ferric chloride $\left(\mathrm{FeCl}_{3} \cdot 6 \mathrm{H}_{2} \mathrm{O}\right)$, ferrous sulfate $\left(\mathrm{FeSO}_{4} \cdot 6 \mathrm{H}_{2} \mathrm{O}\right)$, and ammonia solution (25\%) were purchased from Merck \& Co., Inc. (Kenilworth, NJ, USA). Doxorubicin hydrochlorides were purchased from Sigma-Aldrich Co. (St Louis, MO, USA). Lung carcinoma cells lines (A-549) were purchased from American Type Culture Collection (ATCC, Manassas, VA, USA), and cultured according to instructions supplied by the vendor.

\section{Particle characterization}

\section{Transmission electron microscopy}

Samples for TEM analysis were prepared by drop-coated SPIONs suspension $(10 \mu \mathrm{g} / \mathrm{mL})$ solution. For TEM analysis, the aqueous dispersions were sonicated and drop coated and dried onto formvar-coated 200-mesh copper grids (Ted Pella, Inc., Redding, CA, USA), followed by imaging using a TECNAI G2-30 U TWIN TEM instrument (FEI, Eindhoven, The Netherlands) with an acceleration voltage of $300 \mathrm{kV}$.

\section{Dynamic light scattering}

The size of the nanoparticles was further analyzed by dynamic light scattering measurements, using a NANO-ZS series Malvern Zetasizer instrument (Malvern Instruments, Malvern, UK). Helium-Neon laser (wavelength $633 \mathrm{~nm}$, power $4 \mathrm{~mW}$ ) was used as the light source. The same instrument was used to measure the surface charge (zeta potential) of the nanoparticles.

\section{Hemolysis}

Heparin-stabilized human blood was freshly collected from a healthy volunteer, according to approved Institutional Review Board protocol of University of Delhi. A 4-mL sample of blood was added to $8 \mathrm{~mL}$ of phosphate-buffered saline (PBS), and the red blood cells were isolated from serum by centrifugation at $10,016 \times g$ for 5 minutes.

\section{Cytotoxicity}

We first probed the potential toxicity of these nanoparticles (drug free) in blood, using hemolysis assay. After that, we carried out studies probing the interaction of these nanoparticles, with and without linked drug, using A-549 lung cancer cells in vitro. ${ }^{8}$

\section{Cellular uptake}

After probing the potential toxicity of nondrug-linked nanoparticles in blood and A-549 cells, we began our analysis of interaction of drug-linked nanoparticles (doxorubicin containing citric acid-coated SPION [Dox-CA-SPIONs]) with A-549 cells in vitro. Here, first we monitored the cellular uptake of the drug-linked nanoparticles. ${ }^{9}$
The fixed cells were visualized under a fluorescent Nikon TS-100 inverted microscope, and photographed using a Nikon DIGITAL SIGHT DS-Fi1 Camera (Nikon Corporation, Tokyo, Japan).

\section{Magnetically guided drug delivery}

For analyzing the efficacy of magnetically guided drug targeting of cells using iron oxide nanoparticles, Dox-CA-SPION conjugates were added to cells, with and without a bar magnet placed below the plates. ${ }^{10}$

\section{Results and discussion}

The TEM images of the synthesized citric acid coated SPION (CA-SPION) are shown in Figure 1, showing them to be irregular in size, with an average diameter of $\sim 12 \mathrm{~nm}$.

All samples were prepared in triplicate, and the suspensions were briefly vortexed and centrifuged at $10,016 \times g$ for 5 minutes. Then, $\sim 100 \mu \mathrm{L}$ of supernatant solutions from the sample tube was transferred to a 96-well plate. The absorbance of the supernatant was recorded at $577 \mathrm{~nm}$ (corresponding to hemoglobin absorption), with a reference wavelength of $655 \mathrm{~nm}$. The value of the absorbance at $577 \mathrm{~nm}$ was correlated with the extent of hemolysis. The result of hemolysis assay and cytotoxicity of SPION and CA-SPION are shown in Figure 2A and 2B, respectively.

For analyzing the efficacy of magnetically guided drug targeting of cells using iron oxide nanoparticles, Dox-CA-SPION conjugates were added to cells, with and without a bar magnet placed underneath the plates under magnetic guidance.

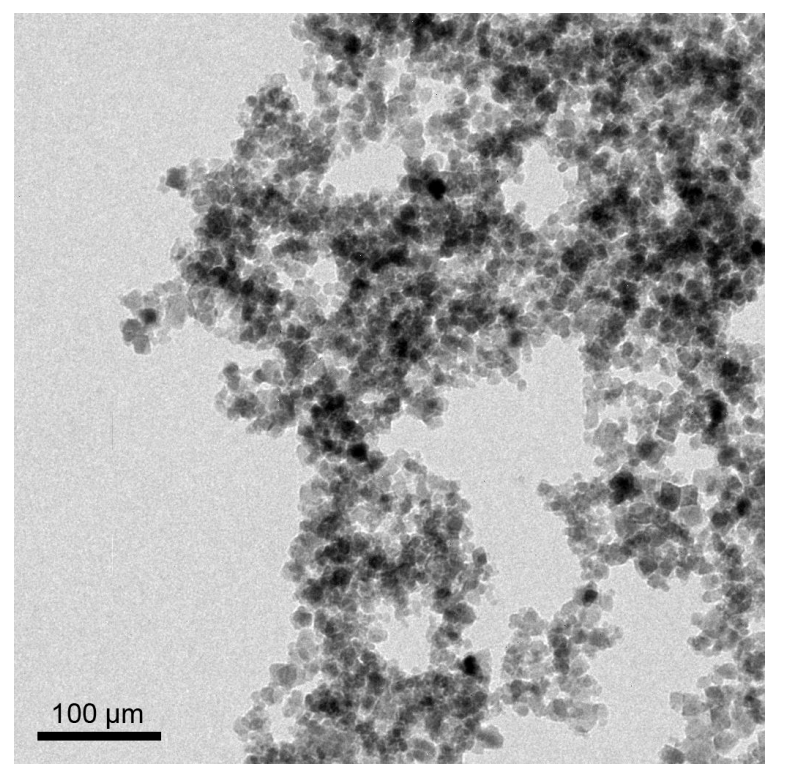

Figure I Transmission electron microscopy pictures of CA-SPION, showing their uniformity of size.

Abbreviation: CA-SPION, citric acid coated superparamagnetic iron oxide nanoparticles. 

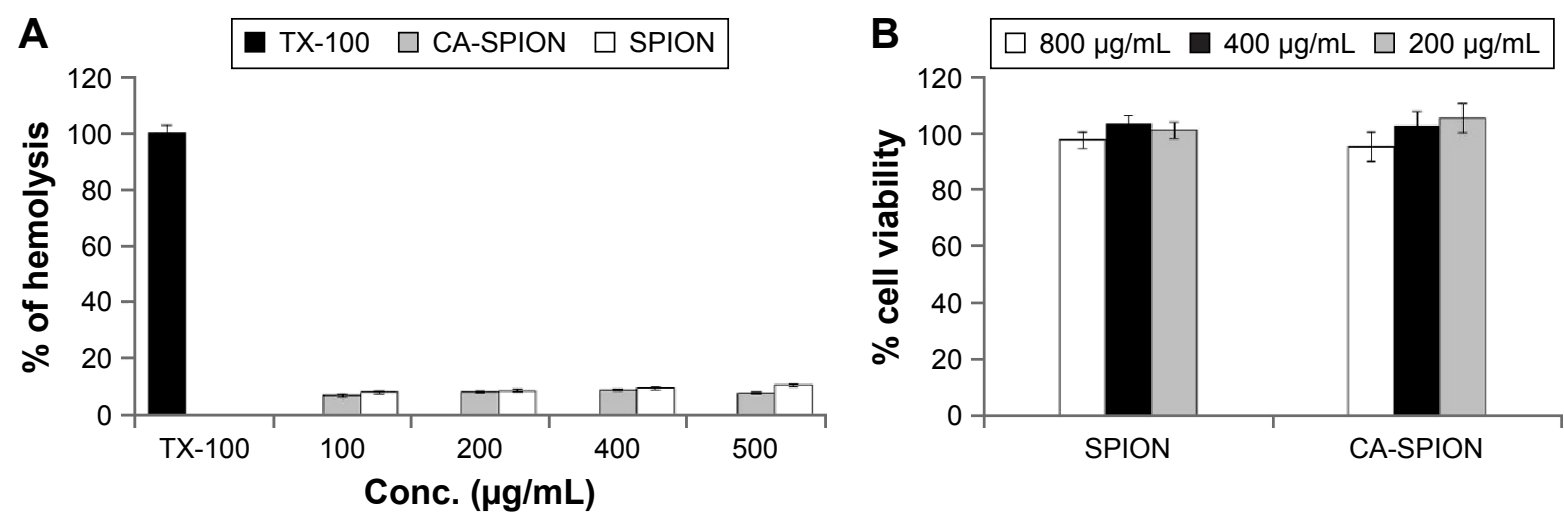

Figure 2 (A) Hemolysis and (B) cell viability result of SPION and CA-SPION.

Abbreviations: SPION, superparamagnetic iron oxide nanoparticles; Conc, concentration; CA-SPION, citric acid coated SPION; TX-100, Triton-X-100.

A total of 12 single-well plates $(35 \mathrm{~mm})$ containing A-549 cells (at a confluence of $\sim 60 \%-70 \%$ ) were treated with $2 \mathrm{mg} / \mathrm{mL}$ of Dox-CA-SPIONs. Magnetic guidance was provided to six plates with nanoparticle-treated cells by placing a bar magnet below them, whereas six remaining plates did not receive magnetic guidance.

After 5 and 15 minutes of incubation, the plates were washed three times with PBS and replenished with $2 \mathrm{~mL}$ of fresh media, followed by 2 hours of incubation. After that, the cells were washed with PBS again and lysed by addition of fixed volume of cell lysis reagent (prepared by dissolving $1 \%$ mass/vol of surfactant Triton-X-100 in PBS, $\mathrm{pH} 7.2$ ).
After 30 minutes, the lysates are transferred to microcentrifuge tubes, centrifuged to separate the cell debris, and the supernatant was analyzed for intracellular iron content and fluorescence of $\operatorname{Dox}\left(\lambda_{\mathrm{ex}}=490 \mathrm{~nm}, \lambda_{\mathrm{em}}=560\right.$ and $\left.590 \mathrm{~nm}\right)$, as shown in Figure $3 \mathrm{~A}$ and B, respectively. Higher intensity of Dox fluorescence coincides with higher nanoparticle uptake of the cells. The cytotoxic effect of Dox-delivery using CASPION under magnetic guidance is shown in Figure 3C.

\section{Conclusion}

In this article, we describe the facile synthesis, characterization, and in vitro applications of drug-conjugated CA-SPION.
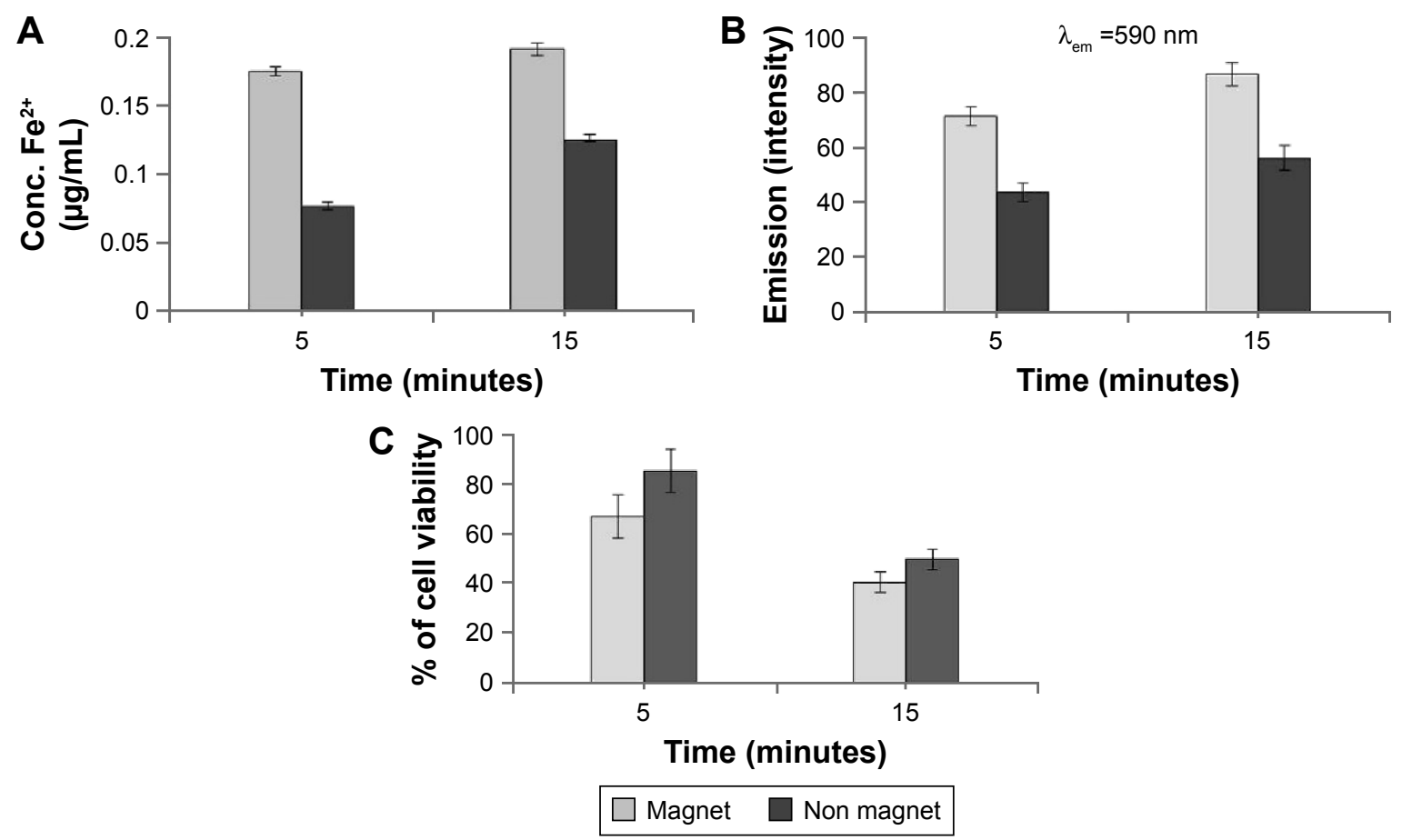

Figure 3 (A) Estimation of total intracellular iron content from cells treated with Dox-CA-SPION, with and without magnetic guidance, (B) estimation of intracellular Doxfluorescence from cells treated with Dox-CA-SPION, and $(\mathbf{C})$ cell viability assay results of DOX-CA-SPION, with and without magnetic guidance. Note: Treatment times were 5 and 15 minutes in each case.

Abbreviations: Conc, concentration; Dox-CA-SPION, doxorubicin containing citric acid-coated SPION; SPION, superparamagnetic iron oxide nanoparticles. 
The conjugated drug releases from the nanoparticles in a sustained manner. The cellular uptake of these nanoparticles was found to be substantial, although it can be further enhanced using magnetic guidance. These nanoparticles (drug free) were found to be nontoxic to cells; however, upon drug conjugation, drug-induced toxicity was observed owing to the slow release of drug from the nanoparticles. The therapeutic efficacy of magnetically targeted drug delivery is expected to be more prominent in in vivo studies, where free drugs are known to be largely ineffective.

\section{Acknowledgment}

We are grateful to University of Delhi, India, for providing a

Research \& Development grant to support this study.

\section{Disclosure}

The authors report no conflicts of interest in this work.

\section{References}

1. Davis SS. Biomedical applications of nanotechnology-implications for drug targeting and gene therapy. Trends Biotechnol. 1997;15:217-224.
2. Li K, Shen M, Zheng L, Zhao J, et al. Magnetic resonance imaging of glioma with novel APTS-coated superparamagnetic iron oxide nanoparticles. Nanoscale Res Lett. 2014;9:304.

3. Sahoo B, Devi KS, Dutta S, et al. Biocompatible mesoporous silicacoated superparamagnetic manganese ferrite nanoparticles for targeted drug delivery and MR imaging applications. J Colloid Interface Sci. 2014;431:31-41.

4. Prasad PN. Introduction to Biophotonics. New York, NY: Wiley; 2004.

5. Kumar LC, Hansel CS, Soboyejo W, et al. LHRH-conjugated magnetic iron oxide nanoparticles for detection of breast cancer metastases. Breast Cancer Res Treat. 2006;99:163-176.

6. Banerjee SS, Chen DH. Multifunctional pH-sensitive magnetic nanoparticles for simultaneous imaging, sensing and targeted intracellular anticancer drug delivery. Nanotechnology. 2008;19(50):505104.

7. Islam MS, Kurawaki J, Kusumoto Y, et al. Hydrothermal novel synthesis of neck-structured hyperthermia-suitable magnetic $\left(\mathrm{Fe}_{3} \mathrm{O}_{4}, \gamma-\mathrm{Fe}_{2} \mathrm{O}_{3}\right.$ and $\alpha-\mathrm{Fe}_{2} \mathrm{O}_{3}$ ) nanoparticles. J Sci Res. 2012;4:97-107.

8. Kumar P, Anuradha, Roy I. Optically and magnetically doped ormosil nanoparticles for bioimaging: synthesis, characterization, and in vitro studies. RSC Adv. 2014;4(4):16181-16187.

9. Roy I, Kumar P, Kumar R, et al. Ormosil nanoparticles as a sustainedrelease drug delivery vehicle. $R S C A d v$. 2014;4(4):53498-53504.

10. Gupta AK, Gupta M. Synthesis and surface engineering of iron oxide nanoparticles for biomedical applications. Biomaterials. 2005;26:3995-4021.

\section{Publish your work in this journal}

The International Journal of Nanomedicine is an international, peerreviewed journal focusing on the application of nanotechnology in diagnostics, therapeutics, and drug delivery systems throughout the biomedical field. This journal is indexed on PubMed Central, MedLine, CAS, SciSearch $\AA$, Current Contents ${ } /$ Clinical Medicine,
Journal Citation Reports/Science Edition, EMBase, Scopus and the Elsevier Bibliographic databases. The manuscript management system is completely online and includes a very quick and fair peer-review system, which is all easy to use. Visit http://www.dovepress.com/ testimonials.php to read real quotes from published authors. 